\title{
Diomega production in relativistic heavy ion collisions
}

\author{
Subrata Pal, ${ }^{1}$ C. M. Ko, ${ }^{1}$ and Z. Y. Zhang ${ }^{2}$ \\ ${ }^{1}$ Cyclotron Institute and Physics Department, Texas A\&M University, College Station, Texas 77843-3366 \\ ${ }^{2}$ Institute of High Energy Physics, 100039 Beijing, People's Republic of China
}

\begin{abstract}
Using a multiphase transport model, we study the production of a new strange dibaryon $(\Omega \Omega)_{0+}$ in dense hadronic matter formed in relativistic heavy ion collisions. The (multi-)strange baryons ( $\Xi$ and $\Omega$ ) are produced by strangeness-exchange reactions between antikaons and hyperons in the pure hadronic phase. The rescattering between the $\Omega$ s at midrapidity leads to a production probability of $\simeq 3 \times 10^{-7}(\Omega \Omega)_{0+}$ per event at the RHIC energy of $\sqrt{s}=130 A \mathrm{GeV}$. The production probability would be enhanced by two orders of magnitude if $(\Omega \Omega)_{0+}$ and $\Omega$ reach chemical equilibrium during heavy ion collisions. We further find that the yield of $(\Omega \Omega)_{0+}$ increases continuously from SPS to the highest RHIC energy.
\end{abstract}

PACS numbers: 25.75.-q, 24.10.Lx, 14.20.Pt

Since first proposed by Jaffe [1] that the $\mathrm{H}$ dibaryon with quark content (uuddss) could be a possible lightest strangelet - droplet of bound strange quark matter, properties of dibaryons have been widely investigated in various models, such as the MIT bag model [2], the Skyrme model [3, 4 , and the constituent quark model [5 8]. The consensus regarding the mass of $\mathrm{H}$ dibaryon is about $2.232 \mathrm{GeV}$ [9]. However, extensive experimental searches [10] have not identified any strangelets with small values of charge fraction, $f_{Z}=|Z| / A<1$, and strangeness fraction $f_{S}=|S| / A<2$.

Recently, the structure and properties of dibaryons with large strangeness are investigated in the chiral $\mathrm{SU}(3)$ model [11 13] that have been quite successful in reproducing several nuclear properties [14]. In this model the $\mathrm{H}$ dibaryon is found to be only weakly bound $[13]$. On the other hand, analysis of some six-quark cluster states with high strangeness fraction reveals that the diomega $(\Omega \Omega)_{0+}$, in particular, is rather deeply bound. Although the color magnetic interaction in the one gluon exchange term for this system exhibits repulsive feature, the large attraction stemming from the chiral quark coupling and from the symmetry property of the system lead to a rather large binding. In the Resonating Group Method calculation, the binding energy of $(\Omega \Omega)_{0+}$ is found to be as large as $\approx 116 \mathrm{MeV}$, and the root mean square distance between the two $\Omega$ 's is $0.84 \mathrm{fm}$. Besides the large (negative) charge fraction $f_{Z}=1$ and strangeness fraction $f_{S}=3$, the new dibaryon $(\Omega \Omega)_{0+}$ has quite a long mean lifetime of $\sim 10^{-10}$ sec as it can undergo only weak decay.

Because of its large strangeness, $(\Omega \Omega)_{0+}$ is not likely to be produced in proton-proton collisions. On the other hand, strangeness production is enhanced in heavy ion collisions and has been suggested as one of the possible signals of quark-gluon plasma (QGP) due to large gluon density and low energy threshold for $s \bar{s}$ formation $[15,16]$. This may thus lead to the formation of exotic deeply bound objects composed of quarks or baryons with large strangeness. Therefore, in relativistic heavy ion collisions, especially at RHIC energies, the dibaryon $(\Omega \Omega)_{0+}$ could be a new interesting candidate.
Indeed, recent measurements by the WA97 Collaboration [17] and the NA49 Collaboration [18] demonstrated substantial enhancement of the (anti-)hyperon yields $(\Lambda$, $\Xi$, and $\Omega$ ) in $158 \mathrm{~A} \mathrm{GeV} \mathrm{Pb}-\mathrm{Pb}$ central collisions relative to $\mathrm{p}-\mathrm{Pb}$ collisions. The enhancement pattern increases with the strangeness content of the (anti-)hyperon. Such a large enhancement for multistrange baryons at midrapidity was interpreted as a signal for quark-gluon plasma formation.

On the other hand, even without a phase transition (anti)strangeness can be abundantly produced by hadron rescatterings alone. In fact, within the microscopic transport model UrQMD, the WA97 data for multistrange baryon enhancement can be explained by reducing the constituent quark mass in the fragmentation of the initial strings in dense matter or by increasing the string tension [19]. Based on the rate equation approach, the multimesonic reactions $\bar{Y}+N \leftrightarrow n \pi+n_{Y} K$ was demonstrated [20] to enhance antistrange hyperon $\bar{Y}$ production in a hadronic scenario. Using a multiphase transport (AMPT) model, we found that strangenessexchange reactions between antikaons and hyperons in a pure hadronic stage also leads to a significant production of multistrange baryons at the SPS and RHIC energies 21].

The considerable production of (multi-)strange baryons in the dense hadronic stage, in absence of QGP formation, could then also result in the formation of exotic objects with enhanced strangeness. Properties of metastable multistrange baryonic objects consisting of nucleons, $\Lambda$ 's and $\Xi$ are studied in the relativistic mean-field theory [22]. These objects were found to have properties quite similar to those of strangelets their quark counterpart. Estimates on the production of such strange dibaryons were presented [23] at the RHIC energies by employing wave-function coalescence in the RQMD model.

The pronounced $\Omega$ production obtained in the AMPT model from strangeness-exchange reactions suggests that multiple collisions between these omegas in the dense hadronic matter may lead to an appreciable production of the new strange dibaryon $(\Omega \Omega)_{0+}$. In this letter, we 
investigate $(\Omega \Omega)_{0+}$ production in relativistic heavy ion collisions in the AMPT model.

The AMPT model 24] is a hybrid model that uses as input both the minijet partons from the hard processes and the strings from the soft processes in the HIJING model [25]. The dynamical evolution of partons are modeled by the ZPC [26] parton cascade model, while the transition from the partonic matter to the hadronic matter is based on the Lund string fragmentation model [27]. The final-state hadronic scatterings are then modeled by the ART model [28]. The AMPT model has been very successful in describing the measured transverse momentum and rapidity distributions of charge particles [29, 30] as well as the particle to antiparticle ratios 31. The multistrange baryon production is via the strangeness-exchange reactions $\bar{K} \Lambda \leftrightarrow \Xi \pi$, $\bar{K} \Sigma \leftrightarrow \Xi \pi$ and $\bar{K} \Xi \leftrightarrow \Omega \pi$. Since there is no experimental information on these cross sections, they are assumed to be the same as that for $\bar{K} N \rightarrow \Sigma \pi$, which is the isospin averaged cross section for converting a nucleon to a sigma [21]. This assumption is consistent with results obtained using $\mathrm{SU}(3)$ invariant hadronic Lagrangians [32]. For diomega production from collisions between omegas, we consider both the the electromagnetic process $\Omega+\Omega \rightarrow(\Omega \Omega)_{0+}+\gamma$ and the strong interaction process $\Omega+\Omega \rightarrow(\Omega \Omega)_{0+}+\eta$. Their cross sections have been evaluated in Ref. [33] using an effective Hamiltonian. For the process $\Omega+\Omega \rightarrow(\Omega \Omega)_{0+}+\gamma$, the maximum cross section is found to be $\approx 1.6 \mu \mathrm{b}$ at $p_{\Omega} \approx 0.5$ $\mathrm{GeV}$, while that for the process $\Omega+\Omega \rightarrow(\Omega \Omega)_{0+}+\eta$, which requires a threshold $p_{\Omega}>0.88 \mathrm{GeV}$ due to finite $\eta$ mass, reaches a maximum value of $\sim 5.5 \mu \mathrm{b}$ at $p_{\Omega} \approx 1.1$ $\mathrm{GeV}$. Because of the mismatch between the large initial and small final relative momenta between the two omegas in the reaction $\Omega+\Omega \rightarrow(\Omega \Omega)_{0+}+\eta$, which involves only strong interactions, its cross section is only slightly larger than that for the reaction $\Omega+\Omega \rightarrow(\Omega \Omega)_{0+}+\gamma$, which involves a much weaker electromagnetic interaction.

To include the diomega production reactions in the AMPT transport model, we parametrize their cross sections as functions of center of mass energy $\sqrt{s}$ (in $\mathrm{GeV}$ ), i.e.,

$$
\sigma_{\Omega+\Omega \rightarrow(\Omega \Omega)+\gamma}=87\left(1-\frac{\sqrt{s}_{0}}{\sqrt{s}}\right)^{0.91}\left(\frac{\sqrt{s}_{0}}{\sqrt{s}}\right)^{24.6} \mu \mathrm{b}
$$

where $\sqrt{s}_{0}=2 m_{\Omega}=3.345 \mathrm{GeV}$, and

$$
\sigma_{\Omega+\Omega \rightarrow(\Omega \Omega)+\eta}=1.5 \times 10^{4}\left(1-\frac{\sqrt{s_{0}}}{\sqrt{s}}\right)^{2}\left(\frac{\sqrt{s_{0}}}{\sqrt{s}}\right)^{34.9} \mu \mathrm{b},
$$

where $\sqrt{s}_{0}=m_{\eta}+2 m_{\Omega}-B_{(\Omega \Omega)}=3.777 \mathrm{GeV}$.

Due to small number of multistrange baryons in heavy ion collisions, the productions of $\Xi$ and $\Omega$ as well as of the diomega are treated perturbatively in the AMPT model [21], i.e., the collision dynamics is assumed to be unaffected by the production of these particles. For $(\Omega \Omega)_{0+}$ production, since its abundance is extremely small, we assume that the production/annihilation rate of $\eta(\gamma)$ is unaffected. Taking a total scattering cross section between two $\Omega$ s to be $\sigma_{\Omega \Omega}=20 \mathrm{mb}$, the two $\Omega \mathrm{s}$ will collide if their distance of closest approach is $\sqrt{\sigma_{\Omega \Omega} / \pi}$. If $P_{\Omega}$ and $P_{\Omega}^{\prime}$ are the production probabilities of the colliding $\Omega \mathrm{s}$, the probability of the resulting diomega is then $P_{(\Omega \Omega)_{0+}}=P_{\Omega} \cdot P_{\Omega}^{\prime} \cdot \Gamma_{\Omega+\Omega \rightarrow(\Omega \Omega)_{0+}}$, where $\Gamma_{\Omega+\Omega \rightarrow(\Omega \Omega)_{0+}}=\left(\sigma_{\Omega+\Omega \rightarrow(\Omega \Omega)+\gamma}+\sigma_{\Omega+\Omega \rightarrow(\Omega \Omega)+\eta}\right) / \sigma_{\Omega \Omega}$. The probabilities of each of the interacting omegas are reduced by $P_{(\Omega \Omega)_{0+}}$. The annihilation cross section of a diomega via the reaction $(\Omega \Omega)_{0+}+\eta \rightarrow \Omega+\Omega$ is obtained using detailed balance of Eq. (2). If a diomega collides with a eta, the diomega is totally annihilated and two omegas are produced with isotropic momentum distribution and each having a probability corresponding to the diomega production probability of $P_{(\Omega \Omega)_{0+}}$. Since diomega production is treated perturbatively, the final results do not depend on the value of $\sigma_{\Omega \Omega}$ used in the AMPT model.

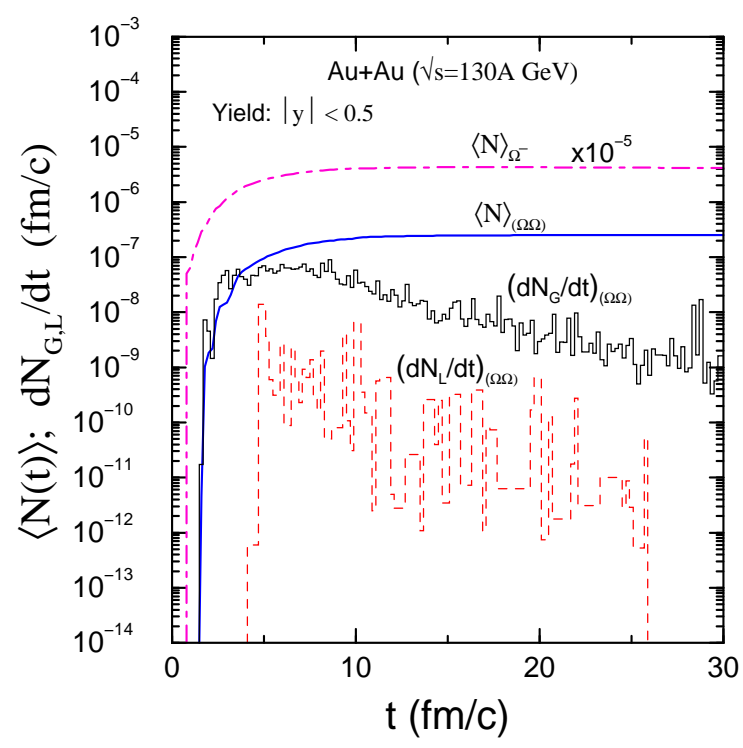

FIG. 1. Time evolution of midrapidity $\Omega^{-}$and $(\Omega \Omega)_{0+}$ for $\mathrm{Au}+\mathrm{Au}$ collisions at the RHIC energy of $\sqrt{s}=130 \mathrm{~A} \mathrm{GeV}$ at an impact parameter of $b \leq 3 \mathrm{fm}$ in the AMPT model. The production and absorption rates for the dibaryon $(\Omega \Omega)_{0+}$ as functions of time is also shown.

In Fig. 1, we show the time evolution of the abundances of midrapidity $\Omega^{-}$and $(\Omega \Omega)_{0+}$ in central $\mathrm{Au}+\mathrm{Au}$ collisions at the RHIC energy of $\sqrt{s}=130 \mathrm{~A} \mathrm{GeV}$. As is evident from the figure, most of the omegas and $(\Omega \Omega)_{0+}$ are produced within $10 \mathrm{fm} / \mathrm{c}$ after hadronization when the energy density is high. Beyond this time the diomega yield saturates to a value of $2.8 \times 10^{-7}$ per event.

From the study of time evolution of the production and absorption rates of particles, we found [21] that most strange hadrons, including the $\Omega \mathrm{s}$, reach chemical equilibrium in collisions at the RHIC energy of $\sqrt{s}=130 \mathrm{~A}$ 
GeV. In Fig. 11 we depict the time evolution of the production (solid lines) and absorption rates (dashed lines) of $(\Omega \Omega)_{0+}$. It is observed that the absorption rate of a diomega by colliding with a eta meson is always smaller compared to its production rate at all times. This stems from additional cross section in the production channel due to the electromagnetic process which has no threshold. We thus infer that the small number of diomega $(\Omega \Omega)_{0+}$ that may be produced in the relativistic collisions at RHIC do not reach chemical equilibrium.

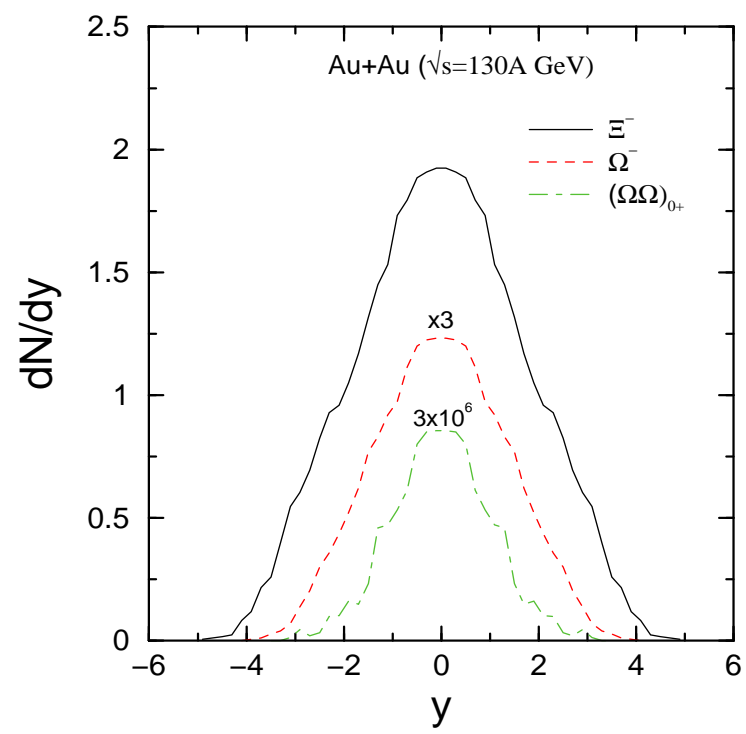

FIG. 2. Rapidity distributions of $\Xi^{-}, \Omega^{-}$, and $(\Omega \Omega)_{0+}$ for $\mathrm{Au}+\mathrm{Au}$ collisions at the RHIC energy of $\sqrt{s}=130 \mathrm{~A} \mathrm{GeV}$ at an impact parameter of $b \leq 3 \mathrm{fm}$ in the AMPT model.

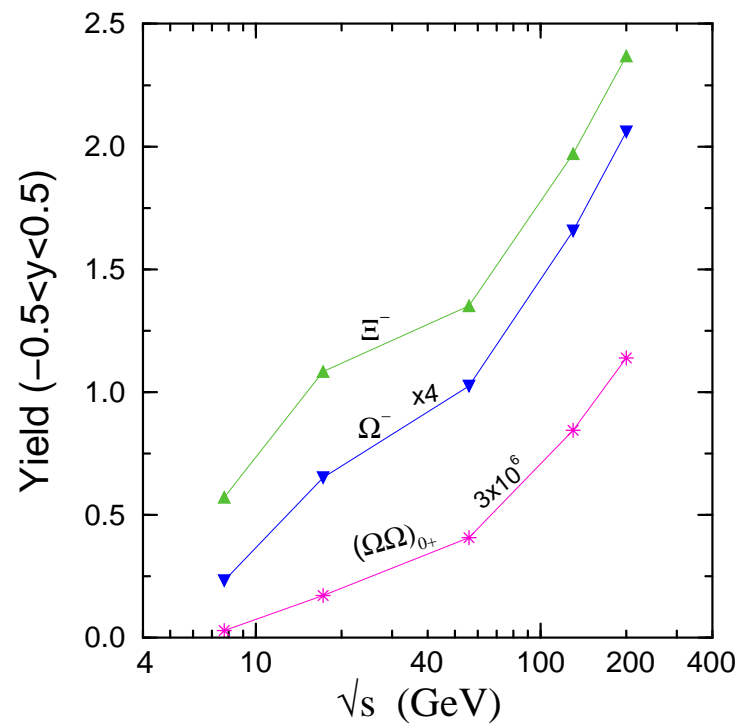

FIG. 3. Energy dependence of $\Xi^{-}, \Omega^{-}$, and $(\Omega \Omega)_{0+}$ at midrapidity $|y|<0.5$ for heavy ion collisions at an impact parameter of $b \leq 3 \mathrm{fm}$ in the AMPT model.
The rapidity distributions of the produced hadrons peak at midrapidity 24 because of increased multiple collisions and rescattering at central rapidities compared to that at large rapidities. In Fig. 2 we show the rapidity distribution of the multistrange particles $\Xi$ and $\Omega$, along with that of $(\Omega \Omega)_{0+}$. The rapidity distribution of particles with increasing strangeness content gradually become narrower as these particles are successively produced from their parent that collide more frequently at small rapidities where the baryon density is high.

We have also studied the energy dependence of diomega production in relativistic heavy ion collisions. In Fig. 3, we show the yield of $(\Omega \Omega)_{0+}$ at midrapidity in $\mathrm{Au}+\mathrm{Au}$ collisions at various energies. Also shown in the figure are the yields of $\Omega^{-}$and $\Xi^{-}$as functions of energies. It is seen that while the $\Omega^{-}$number increases by about a factor of three from the SPS energy $(\sqrt{s}=17 A$ $\mathrm{GeV})$ to the highest RHIC energy $(\sqrt{s}=200 A \mathrm{GeV})$, the $(\Omega \Omega)_{0+}$ number increases by about a factor of 4 , indicating that the dibaryon $(\Omega \Omega)_{0+}$ number reveals a much faster rate of increase with $\sqrt{s}$ as compared to $\Omega^{-}$.

The AMPT model gives a lower bound on the diomega production probability since there might be other production channels, such as $\Omega^{-}+\Xi^{-} \rightarrow(\Omega \Omega)_{0+}+K^{0}$, which have been neglected in the present calculation because of their unknown cross sections. If the diomega production cross section is larger, then diomegas may reach chemical equilibrium with omegas in heavy ion collisions. For most other particles including multistrange baryons, equilibrium thermal models have been successfully employed 34,35 to explain experimental data for their yields and ratios over a wide range of energies from AGS to RHIC. Adopting the statistical model that is based on the grand canonical ensemble with complete thermal, chemical, and strangeness equilibrium, we have found that the results from the AMPT model for the $K^{+} / \pi^{+}, \bar{p} / p$, and $K^{-} / K^{+}$ ratios, which are about $0.18,0.65$, and 0.89 , respectively, at midrapidity in $\mathrm{Au}+\mathrm{Au}$ collisions at energy $\sqrt{\mathrm{s}}=130 \mathrm{~A}$ $\mathrm{GeV}$, can be approximately described with a temperature $T \simeq 170 \mathrm{MeV}$, baryon chemical potential $\mu_{B} \simeq 37 \mathrm{MeV}$, and strange chemical potential $\mu_{S} \simeq 10 \mathrm{MeV}$. If we assume that diomegas are also in chemical equilibrium with omegas, then the ratio $(\Omega \Omega)_{0+} / \Omega^{-}$is $7.4 \times 10^{-5}$. With the omega number of about 0.41 at midrapidity, this leads to a diomega production probability of $\sim 3.0 \times 10^{-5}$ per event, which is two orders of magnitude higher than that obtained in our transport model.

Diomega production in heavy ion collisions can also be studied using the coalescence model [23, 36, 37] based on the omega phase space distribution at freeze out as obtained in the AMPT model. Assuming a harmonic oscillator wave function [37], the Wigner density for the diomega is $\rho_{D}(\mathbf{r}, \mathbf{q})=8 \exp \left(-r^{2} / d^{2}-q^{2} d^{2}\right)$, where $\mathbf{r}=\mathbf{r}_{1}-\mathbf{r}_{2}$ and $\mathbf{q}=\left(\mathbf{p}_{1}-\mathbf{p}_{2}\right) / 2$ are given in the c.m. system of $\Omega-\Omega$, and the value of $d=0.69 \mathrm{fm}$ corresponds to a rms radius of $0.84 \mathrm{fm}$ for the diomega. For central $\mathrm{Au}+\mathrm{Au}$ collisions at $\sqrt{s}=130 \mathrm{~A} \mathrm{GeV}$, the yield of $(\Omega \Omega)_{0+}$ at midrapidity is found to be $2.6 \times 10^{-5}$ and is 
comparable to that from the thermal model. The similar results obtained from both the thermal and coalescence models are not surprising as it was shown in Ref. [38], that the two models are equivalent when matter is in thermal and chemical equilibrium and the binding energy of the composite particle is much smaller than the temperature. Since the AMPT model predicts a hadronic matter at freeze out that is close to thermal and chemical equilibrium, the diomega yield from the coalescence model thus should be similar to that given by the thermal model.

Our estimate for the production probability of $(\Omega \Omega)_{0+}$ are well within the limits of the present detectors used at RHIC energies. Therefore, this exotic object can, in principle, be detected in present and future experiments. The fact that $(\Omega \Omega)_{0+}$ has a large strangeness content with a binding energy of $\simeq 116 \mathrm{MeV}$, it is stable against strong hadronic decays and possess weak decays: $(\Omega \Omega)_{0+} \rightarrow \pi^{-}+\Xi^{0}+\Omega^{-}$and $(\Omega \Omega)_{0+} \rightarrow \pi^{0}+\Xi^{-}+\Omega^{-}$. Because three-body decay are involved, the final state phase-space would be suppressed. In the sudden approximation, the mean lifetime of $(\Omega \Omega)_{0+}$ was found [12] to be about four times longer than the free $\Omega$ lifetime of $0.822 \times 10^{-10}$ sec. Apart from these conventional decay modes, the nonmesonic decay $(\Omega \Omega)_{0+} \rightarrow \Xi^{-}+\Omega^{-}$is also possible; the estimated [12] lifetime of $(\Omega \Omega)_{0+}$ for this process is twice the free $\Omega$ lifetime. Thus, instead of direct observation, the $(\Omega \Omega)_{0+}$ may also be detected in the $\Xi^{-} \Omega^{-}$invariant mass distribution. The observation of which could provide useful information of the unknown $\Omega-\Omega$ interaction strength. Our study thus opens up the intriguing possibility of detecting the new dibaryon $(\Omega \Omega)_{0+}$ in heavy ion collisions at the RHIC energies.

This work of SP and CMK is supported by the National Science Foundation under Grant No. PHY-9870038, the Welch Foundation under Grant No. A-1358, and the Texas Advanced Research Program under Grant No. FY99-010366-0081, while that of ZYZ is supported by the National Natural Science Foundation of China and the Chinese Academy of Sciences.

[1] R.L. Jaffe, Phys. Rev. Lett. 38, 195 (1977).

[2] A. Chodos, et al., Phys. Rev. D 9, 3471 (1974); T. DeGrand, et al., Phys. Rev. D 12, 2060 (1975).

[3] G.S. Adkins et al., Nucl. Phys. B228, 552 (1983).

[4] E.M. Nyman, Phys. Lett. B 142, 388 (1984).

[5] M. Oka and K. Yazaki, Phys. Lett. B 90, 41 (1980).

[6] K. Yazaki, Prog. Theor. Phys. Suppl. 91, 146 (1987).

[7] A. Valcarce, B. Buchmann, A. Fernandez and A. Faessler,
Phys. Rev. C 50, 2246 (1994).

[8] K. Shimizu, M. Koyama, Nucl. Phys. A646, 211 (1999).

[9] C.B. Dover, Nucl. Phys. A450, 95c (1986); C.B. Dover, P. Koch and M. May, Phys. Rev. C 40, 115 (1989).

[10] K. Imai, in Hadrons and Nuclei with Strangeness, Proceedings of the First Sino-Japan Symposium on Strangeness Physics (1999).

[11] Z.Y. Zhang, Y.W. Yu and X.Q. Yuan, Nucl. Phys. A670, $178 c$ (2000).

[12] Z.Y. Zhang, Y.W. Yu, C.R. Ching, T.H. Ho and Z.D. Lu, Phys. Rev. C 61, 065204 (2000).

[13] Q.B. Li, P.N. Shen, Z.Y. Zhang and Y.W. Yu, Nucl. Phys. A683, 487 (2001).

[14] Z.Y. Zhang, Y.W. Yu, P.N. Shen and L.R. Dai, Nucl. Phys. A625, 59 (1997).

[15] J. Rafelski and B. Müller, Phys. Lett. B 101, 111 (1982).

[16] P. Koch, B. Müller, and J. Rafelski, Phys. Rep. 142, 67 (1986).

[17] E. Anderson et al., (WA97 Collaboration), Phys. Lett. B 433, 209 (1998); ibid. 449, 401 (1999).

[18] S. Margetis et al., (NA49 Collaboration), J. Phys. G 25, 189 (1999); F. Gabler et al., (NA49 Collaboration), J. Phys. G 25, 199 (1999).

[19] S. Soff, S.A. Bass, M. Bleicher, L. Bravina, E. Zabrodin, H. Stöcker, and W. Greiner, Phys. Lett. B 471, 89 (1999).

[20] C. Greiner and S. Leupold, nucl-th/0009036.

[21] S. Pal, C.M. Ko and Z. Lin, nucl-th/0106073.

[22] J. Schaffner-Bielich, C. Greiner, A. Diener and H. Stöcker, Phys. Rev. C 55, 3038 (1997).

[23] J. Schaffner-Bielich, R. Mattiello and H. Sorge, Phys. Rev. Lett. 84, 4305 (2000).

[24] B. Zhang, C.M. Ko, B.A. Li and Z. Lin, Phys. Rev. C 61, 067901 (2000); B. Zhang, C.M. Ko, B.A. Li, Z. Lin, and B.H. Sa, ibid. 62, 054905 (2000); Z. Lin, S. Pal, C.M. Ko, B.A. Li and B. Zhang, ibid. 64, 011902 (2001).

[25] M. Gyulassy and X.N. Wang, Comp. Phys. Comm. 83, 307 (1994).

[26] B. Zhang, Comp. Phys. Comm. 109, 193 (1994).

[27] T. Sjöstrand, Comp. Phys. Comm. 82, 74 (1994).

[28] B.A. Li and C.M. Ko, Phys. Rev. C 52, 2037 (1995).

[29] B.B. Back et al., Phys. Rev. Lett. 85, 3100 (2000).

[30] K.H. Ackermann et al., Phys. Rev. Lett. 86, 402 (2001).

[31] B.B. Back et al., nucl-exp/0105011.

[32] C.H. Li, C.M. Ko, and Z.W. Lin, to be published.

[33] Y.W. Yu, P. Wang, Z.Y. Zhang, C.R. Ching and T.H. Ho, Comm. Theo. Phys. 35, 553 (2001).

[34] P. Braun-Munzinger, J. Stachel, J.P. Wessels and N. Xu, Phys. Lett. B 344, 43 (1995); ibid 365, 1 (1996); P. Braun-Munzinger, I. Heppe and J. Stachel, Phys. Lett. B 465, 15 (1999); P. Braun-Munzinger, D. Magestro, K. Redlich, and J. Stachel, hep-ph/0105229.

[35] J. Cleymans and K. Redlich, Phys. Rev. C 60, 054908 (1999).

[36] H. Sato and K. Yazaki, Phys. Lett. B 98, 153 (1981).

[37] M. Gyulassy, K. Frankel, and E.A. Remler, Nucl. Phys. A402, 596 (1983).

[38] A. Mekjian, Phys. Rev. Lett. 38, 640 (1977). 